\title{
Prognostic impact of SET domain-containing protein 8 and protein arginine methyltransferase 5 in patients with hepatocellular carcinoma following curative resection
}

\author{
ZHIFEI LIN $^{1 *}$, HULIANG JIA ${ }^{1 *}$, LIANG HONG $^{2 *}$, YAHUI ZHENG ${ }^{3}$, WEIQIN SHAO ${ }^{1}$, XUDONG REN $^{1}$, \\ WENWEI ZHU ${ }^{1}$, LU LU ${ }^{1}$, MING LU ${ }^{1}$, JUBO ZHANG ${ }^{3}$ and JINHONG CHEN ${ }^{1}$ \\ ${ }^{1}$ Department of General Surgery, Huashan Hospital, Fudan University, Shanghai 200040; \\ ${ }^{2}$ Department of Infectious Diseases, Ruian People's Hospital, Wenzhou, Zhejiang 325200; \\ ${ }^{3}$ Department of Infectious Diseases, Huashan Hospital, Fudan University, Shanghai 200040, P.R. China
}

Received December 13,2017; Accepted April 4, 2018

DOI: $10.3892 / \mathrm{ol} .2018 .9083$

\begin{abstract}
Histone methyltransferases are important determinants of the initiation and progression of hepatocellular carcinoma (HCC) and represent promising therapeutic targets. However, whether the expression profile of multiple histone methyltransferases represents a poorer prognosis is entirely unknown. The aim of the present study was to investigate the association between histone methylation and HCC phenotype, and the prognostic value of combining expression levels of SET domain-containing protein 8 (SET8) with protein arginine methyltransferase 5 (PRMT5) in patients with HCC following curative resection. The retrospective study included 195 consecutive patients who had undergone hepatectomy for HCC. Immunohistochemical staining for SET8 and PRMT5 was performed on paraffin-embedded tumor
\end{abstract}

Correspondence to: Dr Jubo Zhang, Department of Infectious Diseases, Huashan Hospital, Fudan University, 12 Middle Urumqi Road, Shanghai 200040, P.R. China

E-mail: drzhangjubo@163.com

Professor Jinhong Chen, Department of General Surgery, Huashan Hospital, Fudan University, 12 Middle Urumqi Road, Shanghai 200040, P.R. China

E-mail: jinhongch@hotmail.com

*Contributed equally

Abbreviations: HCC, hepatocellular carcinoma; SET8, SET domain-containing protein 8; PRMT5, protein arginine methyltransferase 5; ER, early intrahepatic recurrence; TNM, Tumor-Node-Metastasis; TMA, tissue microarray; DAB, 3,3'-diaminobenzidine; OS, overall survival; TTR, time to recurrence; ROC, receiver operating characteristic; CI, confidence interval; HR, hazard ratio; NA, not adopted; NS, not significant; AFP, $\alpha$-fetoprotein; UICC, International Union Against Cancer

Key words: SET domain-containing protein 8, protein arginine methyltransferase 5 , hepatocellular carcinoma, recurrence, prognosis tissue microarrays. Expression was analyzed for correlations with clinicopathological features, marker co-expression and patients' survival by univariate and multivariate analyses. Positive SET8 expression was noted in 104 patients $(53.3 \%)$, and was associated with PRMT5 expression $(n=106,54.4 \%$, $\mathrm{P}<0.05)$. Immunohistochemical analysis demonstrated that high expression of SET8 and PRMT5 was significantly associated with poor overall survival $(\mathrm{OS}, \mathrm{P}<0.001)$ and time to recurrence (TTR, $\mathrm{P}<0.001)$. Multivariate Cox analysis revealed that SET8 and PRMT5, along with vascular invasion, tumor size and tumor number, were independent prognostic factors for OS and TTR. The combination of SET8 and PRMT5 demonstrated an improved capacity to predict patient mortality and disease recurrence $(\mathrm{P}=0.002$ and $\mathrm{P}=0.004$, respectively), particularly for the prediction of early recurrence $(\mathrm{P}<0.001)$. In conclusion, high expression of SET8 combined with PRMT5 was associated with a high rate of recurrence and poor survival in patients with HCC. The independent pattern of histone methylation represents a novel insight into tumor progression and therapeutic targets for HCC.

\section{Introduction}

The initiation and progression of hepatocellular carcinoma (HCC) is regarded as a multi-step process entailing genetic and epigenetic alterations (1-3). Epigenetic modifications can affect gene expression at the cancer transcriptome level more extensively than genetic changes. Furthermore, the majority of epigenetic alterations are reversible, and therefore could provide a prospective treatment for HCC (4). Increasing evidence has suggested that tumor-associated histone methylation is an important determinant of initiation and the development of HCC, and represents a promising therapeutic target (5).

Histone methylation can be divided into arginine methylation and lysine methylation. SET domain-containing protein 8 (SET8) is a member of the SET domain-containing methyltransferase family, which particularly catalyzes the monomethylation of histone H4 Lys20 (6-8). SET8 has been reported to participate in diverse biological processes, the 
regulation of gene transcription, maintenance of genomic stability, formation of heterochromatin, and cellcycle progression and development (9-15). Protein arginine methyltransferase 5 (PRMT5), a type II arginine methyltransferase, regulates gene transcription and modulates chromatin structure through symmetrically dimethylating arginine residues on target proteins $(16,17)$. H3R8 and H4R3 are the key repressive histone methylation sites that are methylated by PRMT5 $(18,19)$. Accumulating evidence has suggested that PRMT5 may act as an oncogene in multiple tumors via epigenetic repression of several tumor suppressor genes or by post-translational modification of signaling molecules $(16,20,21)$.

Histone methylation has diverse functional consequences depending on the type of residue and which site the methylation modifies (22). Several studies have revealed a complex interplay between the distinct covalent modifications that occur on the histone tails, including methylation of histone $\mathrm{H} 3$ and $\mathrm{H} 4$, which supported the histone code hypothesis $(23,24)$. The patterns of histone methylation associated with progression of cancer have potential clinical value. The present study revealed that there was a significant correlation between distinct histone methylations, and their combination to predict survival and tumor recurrence, particularly for the prediction of early recurrence (ER).

\section{Patients and methods}

Patients and HCC tissue. A total of 195 HCC patients (175 male and 20 female) who underwent curative resection by the same surgical team between 1999 and 2006 at the Liver Cancer Institute of Fudan University (Shanghai, China) were included in this study. The mean age of patients was 52 years (range, 26-78 years). The surgical techniques for liver resection have been described previously (25). The inclusion and exclusion criterion of patient cohort include (1) having a distinctive pathologic diagnosis of $\mathrm{HCC},(2)$ without preoperative anticancer treatment and extrahepatic metastases, (3) under primary and curative liver resection, and (4) with complete clinicopathologic and follow-up data. Tumor tissue was collected with the written informed consent of each patient and the study was approved by the Ethics Committee of Zhongshan Hospital.

Patients in the cohort were followed up until March 2012 , with a median observation time of 60 months (range, 2-142 months). The follow-up procedures were described in a previous study (25), and treatment programs following relapse were administered according to a uniform guideline (26). Tumor staging was determined according to the 2002 International Union Against Cancer Tumor-Node-Metastasis (TNM) classification system. Overall survival (OS) was defined as the interval between the dates of surgery and patient mortality. Time to recurrence (TTR) was defined as the interval between the dates of surgery and the first detection of intrahepatic or distant recurrence. Early recurrence was defined as any recurrence type that occurred within the time period of up to and including 24 months (27). Data were censored at the final follow-up for patients without recurrence or mortality.

Tissue microarray (TMA) and immunohistochemistry. Tissue microarrays were conducted as described previously (25), and were embedded by Shanghai Biochip Company Ltd. (Shanghai, China). Immunohistochemistry for SET8 and PRMT5 was performed as described previously (25). Briefly, sections were deparaffinized in xylene $(100 \%$, three times, 5 min each) and rehydrated by stepwise washes in decreasing ethanol solutions ratio (100, 95, 80 and $75 \%$ for 5 min each) at room temperature. Slides were treated with $3 \%$ hydrogen peroxide for $15 \mathrm{~min}$ at room temperature. Antigen retrieval was achieved by boiling slides in the autoclave $\left(80 \mathrm{kpa}\right.$ and $\left.100^{\circ} \mathrm{C}\right)$ for $1 \mathrm{~min}$ in $0.01 \mathrm{M}$ sodium citrate buffer ( $\mathrm{pH} \mathrm{6.0),} \mathrm{followed}$ by cooling to room temperature. Sections were incubated with $10 \%$ normal goat serum (Boster Biological Technology, Pleasanton, CA, USA) for $60 \mathrm{~min}$ at room temperature to block nonspecific antigen sites. Slides were incubated with primary antibodies against SET8 (1:200 dilution; Cell Signaling Technology, Inc., Danvers, MA, USA) or PRMT5 (1:200 dilution; Abcam, Cambridge, UK) overnight at $4^{\circ} \mathrm{C}$, followed by incubation with a biotin-conjugated secondary antibody, and horseradish peroxidase-conjugated streptavidin for $60 \mathrm{~min}$ at room temperature. The sections were stained with DAB for $1 \mathrm{~min}$ at room temperature and counterstained with hematoxylin for $1 \mathrm{~min}$ at room temperature. Negative controls were treated identically but with the primary antibody omitted.

The density of positive staining was measured with a computerized image system. Briefly, images of the representative fields were captured and analyzed with Image-Pro Plus version 6.2 software (Media Cybernetics, Inc., Rockville, MD, USA). The integrated optical density of the positive staining for SET8 and PRMT5 was measured, and the ratio to total area of each image was defined as the SET8 and PRMT5 density. The final staining score was classified into four grades: Score 1 (weak, less than the 25 th percentile value), score 2 (moderate, between 25th and 50th percentile value), score 3 (strong, between 50th and 75th percentile value), and score 4 (very strong, higher than 75th percentile value). HCC tissues with staining scores 1 and 2 were defined as low expression, and those with scores 3 and 4 were defined as high expression (representative figures are demonstrated in Fig. 1).

Statistical analysis. Statistical analysis was performed with SPSS 13.0 for Windows (SPSS Inc., Chicago, IL, USA). The mean, standard deviation and frequency were used to summarize patient variables within the SET8/PRMT5 high and low expression groups. The Pearson's $\chi^{2}$ test or Fisher's exact test were used to compare categorical variables; and continuous variables were analyzed by the Students t-test or Pearson's correlation test. SET8 and PRMT5 expression in tumor tissues was analyzed using the Spearman's rank correlation test. Kaplan-Meier analysis was used to determine OS. The log-rank test was used to compare OS between subgroups and the Cox regression model was used to perform multivariate analyses. Receiver operating characteristic (ROC) curve analysis was used to determine the predictive value of the parameters. $\mathrm{P}<0.05$ was considered to indicate a statistically significant difference.

\section{Results}

Clinicopathological data. The present study included 195 patients with surgically resected TNM stage I-IIIA 
A

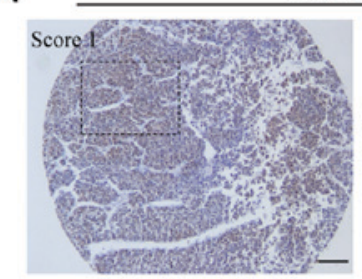

Low Set8
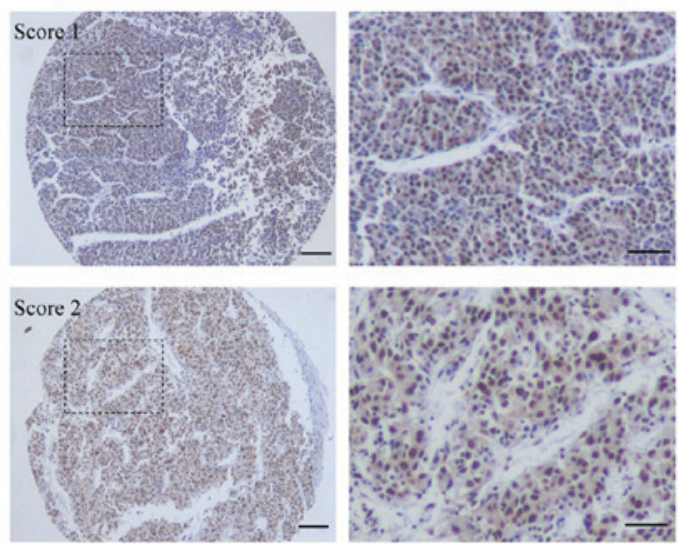

B

$\sum_{\frac{x}{a}}^{\circ}$

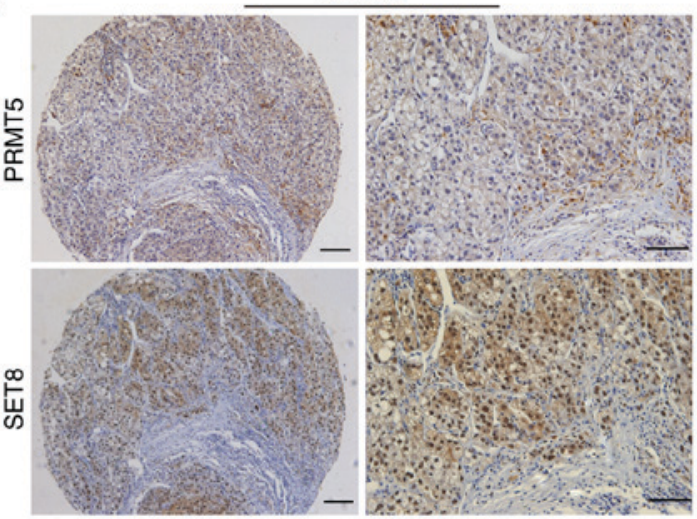

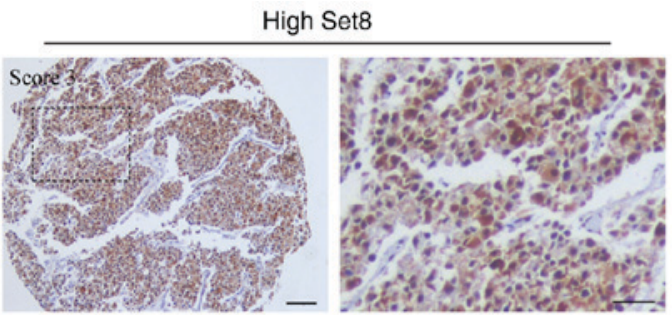
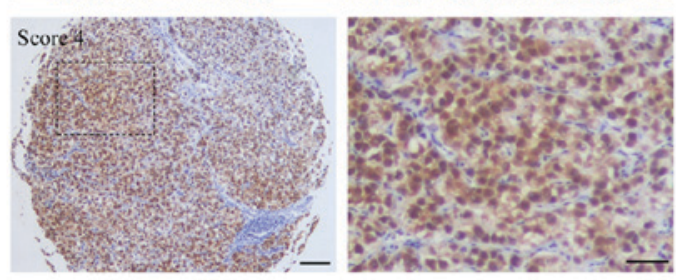

Case 39

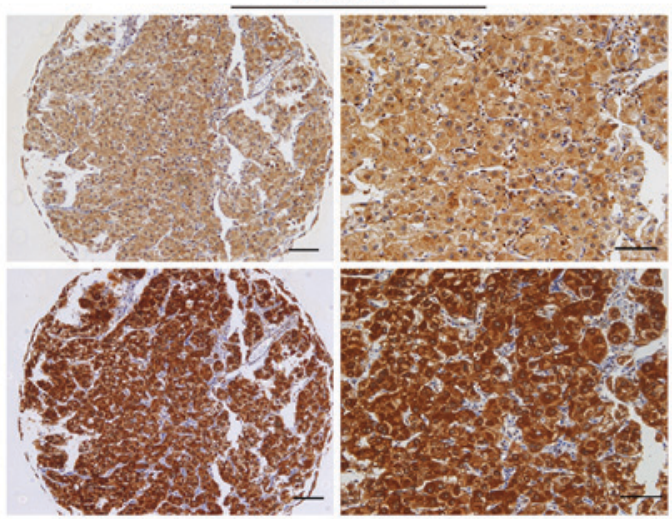

Figure 1. Expression of, and association between, SET8 and PRMT5 in HCC tissue arrays. (A) Representative immunohistochemical staining of SET8 are (magnification, x100; scale bar, $100 \mu \mathrm{m}$ ), along with corresponding x400 magnification images (scale bar, $50 \mu \mathrm{m}$ ). The intensity of the nuclear expression of SET8 in HCC cells was graded as low (scores 1 and 2) or high (scores 3 and 4). (B) Consecutive sections of two clinical cases were used for immunohistochemical staining of SET8 and PRMT5. SET8, SET domain-containing protein 8; PRMT5, protein arginine methyltransferase 5; HCC, hepatocellular carcinoma.

HCC; comprising 123 stage I, 31 stage II and 41 stage IIIA patients. At the time of the last follow-up, mortality was noted in 126 patients, including 11 patients who succumbed to liver failure or other diseases without record of tumor recurrence; and 148 patients exhibited tumor recurrence. Treatment programs following relapse included resection $(n=36)$, trans-arterial chemoembolization $(n=79, x 1-10)$, radiofrequency ablation and percutaneous ethanol injection $(n=55)$ and radiotherapy $(n=6)$ according to a uniform guideline. Among recurrent patients, six were unable to tolerate any antitumor treatments due to severe liver dysfunction or weak general performance. The 1-, 3- and 5-year OS rates were 79.8,62.3 and 47.5\%, respectively, and the 1-, 3- and 5-year probabilities of recurrence were 27.4, 57.2 and $67.2 \%$, respectively.

Expression of SET8 and PRMT5, and their correlation with clinicopathological characteristics in HCC. Immunohistochemical analysis revealed that the staining of SET8 was mainly limited to the nucleus, whereas the expression pattern of PRMT5 in the tumor cells was either diffuse in the cytoplasm or concentrated in the nucleus. Representative low staining and high staining of SET8 in HCC tissues are presented in Fig. 1A. According to the staining scores, 53.3\% of HCC samples (104/195) revealed a high expression of SET8, and $54.4 \%$ of HCC samples (106/195) revealed a high expression of PRMT5 (data not shown). As is presented Fig. 1B, low expression of SET8 is associated with low PRMT5 in the same HCC specimen, and high expression of SET8 is also correlated with high PRMT5 in the same HCC specimen. In addition, a statistically significant positive correlation between SET8 and PRMT5 expression in tumor tissue was observed $(\mathrm{P}<0.05$; Fig. 2).

The present study assessed whether SET8 and PRMT5 expression were correlated with clinicopathological parameters, including age, sex, Eastern Cooperative Oncology Group (ECOG) performance score, liver cirrhosis, serum AFP level, encapsulation, vascular invasion, tumor size, tumor number and TNM stage. It was identified that SET8 and PRMT5 expression was not statistically correlated with any clinicopathological characteristics (data not shown).

Prognostic significance of the expression of SET8 and PRMT5. Kaplan-Meier survival curves revealed that high expression of SET8 or PRMT5 was significantly associated with a lower OS rate $(\mathrm{P}=0.001$ and $\mathrm{P}=0.006$, respectively; Fig. $3 \mathrm{~A})$ and $\mathrm{TTR}$ $(\mathrm{P}<0.001$ and $\mathrm{P}=0.002$, respectively; Fig. 3A). In univariate analysis, several clinicopathological factors, including AFP, vascular invasion, tumor size, tumor number and TNM stage revealed prognostic significance for OS and TTR $(\mathrm{P}<0.05$ for all, Table I). Liver cirrhosis was also associated with OS ( $\mathrm{P}=0.038$, Table I). In addition, all clinicopathological factors revealing significance by univariate analysis, with the 


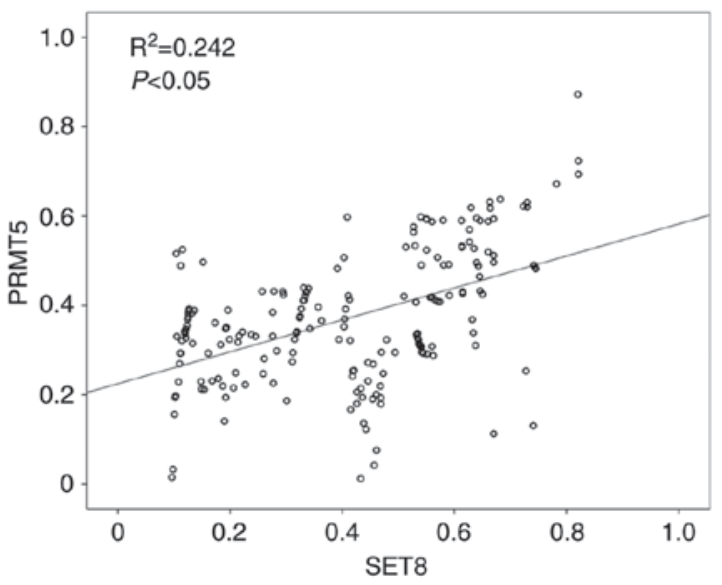

Figure 2. Correlation between SET8 and PRMT5 expression in hepatocellular carcinoma. Scatterplot of SET8 versus PRMT5 with regression line. $\mathrm{P}<0.05$, Spearman's correlation coefficient. SET8, SET domain-containing protein 8; PRMT5, protein arginine methyltransferase 5.

exception of TNM stage, were adopted when multivariate Cox proportional hazards analysis was performed (Table I). The results suggested that vascular invasion, tumor size, tumor number, and SET8 and PRMT5, were independent risk factors for OS and TTR $(\mathrm{P}<0.05$ for all, Table I).

Combination of SET8 and PRMT5 expression and ROC analysis. Patients were classified into three groups according to their SET8 and PRMT5 expression: Group I $(n=44)$, low expression of SET8 and PRMT5; group II ( $n=92)$, high expression of SET8 or PRMT5; and group III $(n=59)$, high expression of SET8 and PRMT5. Differences in OS $(\mathrm{P}<0.001)$ and TTR $(\mathrm{P}<0.001)$ were significant among the three groups. The 5-year OS and TTR rates were 67.1 and $55.3 \%$, respectively, for group I, but only 27.5 and $18.3 \%$, respectively, for group III (Fig. 3B).

Clinicopathological variables that revealed a significant result in multivariate survival analyses and in combination with SET8 and PRMT5 were incorporated in ROC analyses. All the incorporated variables were significantly associated with patient mortality and disease recurrence $(\mathrm{P}<0.05)$. The predictive value of SET8 combined with PRMT5 for OS and TTR was revealed to be more accurate than SET8 or PRMT5 alone. For patient mortality and disease recurrence, the predictive value of the combination of SET8 and PRMT5 was the most accurate (Fig. 3C). The area under the curve of this combination was 0.635 [95\% confidence interval (CI), 0.554-0.716; $\mathrm{P}=0.002$ ] for patient mortality and 0.641 (95\% CI, $0.552-0.730 ; \mathrm{P}=0.004$ ) for disease recurrence.

Combination of SET8 and PRMT5 in predicting the prognosis of early stage HCC. Due to the high rates of recurrence following hepatectomy, it is critical to identify reliable prognostic markers to define patients at high risk of recurrence, particularly for patients with early stage disease who do not have significant vascular invasion or regional or distant metastasis. To fully determine and validate the prognostic value of the combination of SET8 and PRMT5 in patients with HCC, particularly for those with early stage HCC, the prognostic value of the combination of SET8 and PRMT5 in the
TNM stage I subgroup was further investigated $(n=123)$. The combination of SET8 and PRMT5 was demonstrated to be a risk factor associated with $\mathrm{OS}(\mathrm{P}=0.012)$ and TTR $(\mathrm{P}=0.006$; Fig. 4). The median survival time was 45 months and mortality was observed in 24/34 patients. A high expression of SET8 and PRMT5 was correlated with a median survival time of $>116$ months. Mortality in 12/28 patients was correlated with a low expression of SET8 and PRMT5. Furthermore, stratified analyses according to serum AFP levels were also conducted to investigate the prognostic value of individual expression levels of SET8 and PRMT5 and their combination. Of note, in the lower AFP subgroup ( $\leq 400 \mathrm{ng} / \mathrm{ml})$, individual expression levels of SET8 and PRMT5 and their combination were significantly associated with OS and TTR in the subgroups $(\mathrm{P}<0.05$, data not shown $)$

The prognostic value of SET8 and PRMT5 for ER. Recurrence, particularly early intrahepatic recurrence (ER) within 2 years after hepatectomy, is the main cause for the poor prognosis associated with HCC (28). The prognosis of early intrahepatic recurrence is much worse than that of late intrahepatic recurrence, due to the multi-centric occurrence of a new tumor. In the present study, all the recurrences were divided into early and late groups, with 24 months as the cut-off value, and the prognostic value of SET8 and PRMT5 in the ER subgroup was investigated. The results revealed that the patients with a high SET8 or PRMT5 expression tended to have an ER $(\mathrm{P}<0.001$ and $\mathrm{P}=0.006$, respectively, Fig. $5 \mathrm{~A}$ and $\mathrm{B}$ ). The patients with both high expression of SET8 and PRMT5 in ER sub group III showed a higher risk of early recurrence (Fig. 5C). When SET8 and PRMT5 were further combined, significant differences between high and low combination of the two markers were reached (Fig. 5D). The area under the curve was up to 0.660 (95\%CI, 0.584-0.736; $\mathrm{P}<0.001)$, which was the largest among the adopted factors (data not shown).

\section{Discussion}

Recurrence following hepatectomy remains the major obstacle for further improving the outcomes of HCC (26). Currently, there are no effective therapeutic strategies that can reduce recurrence as it is difficult to determine which patients will have tumor recurrence following hepatectomy. Global histone modification patterns can predict the risk of cancer recurrence and clinical outcome, representing a novel insight into therapeutic targets for cancer (29). As histone methylations are important determinants in the progression of HCC, identification of biomarkers that focus on multiple histone methylation in tumor tissue may aid in predicting the prognosis and recurrence of HCC. In the present study, it was revealed that SET8 combined with PRMT5 was a promising predictor of OS and disease recurrence in HCC, particularly for those with early stage HCC or low AFP levels.

The study of potential prognostic markers in large numbers of clinical specimens is crucial in translating novel findings from basic science into clinical practice. The 'histone code' hypothesis proposes that distinct combinations of histone modifications can generate unique transcriptional functions $(23,30)$. A previous study suggested that different $\mathrm{H} 3$ and $\mathrm{H} 4$ tail modifications can act sequentially or in combination to mediate 

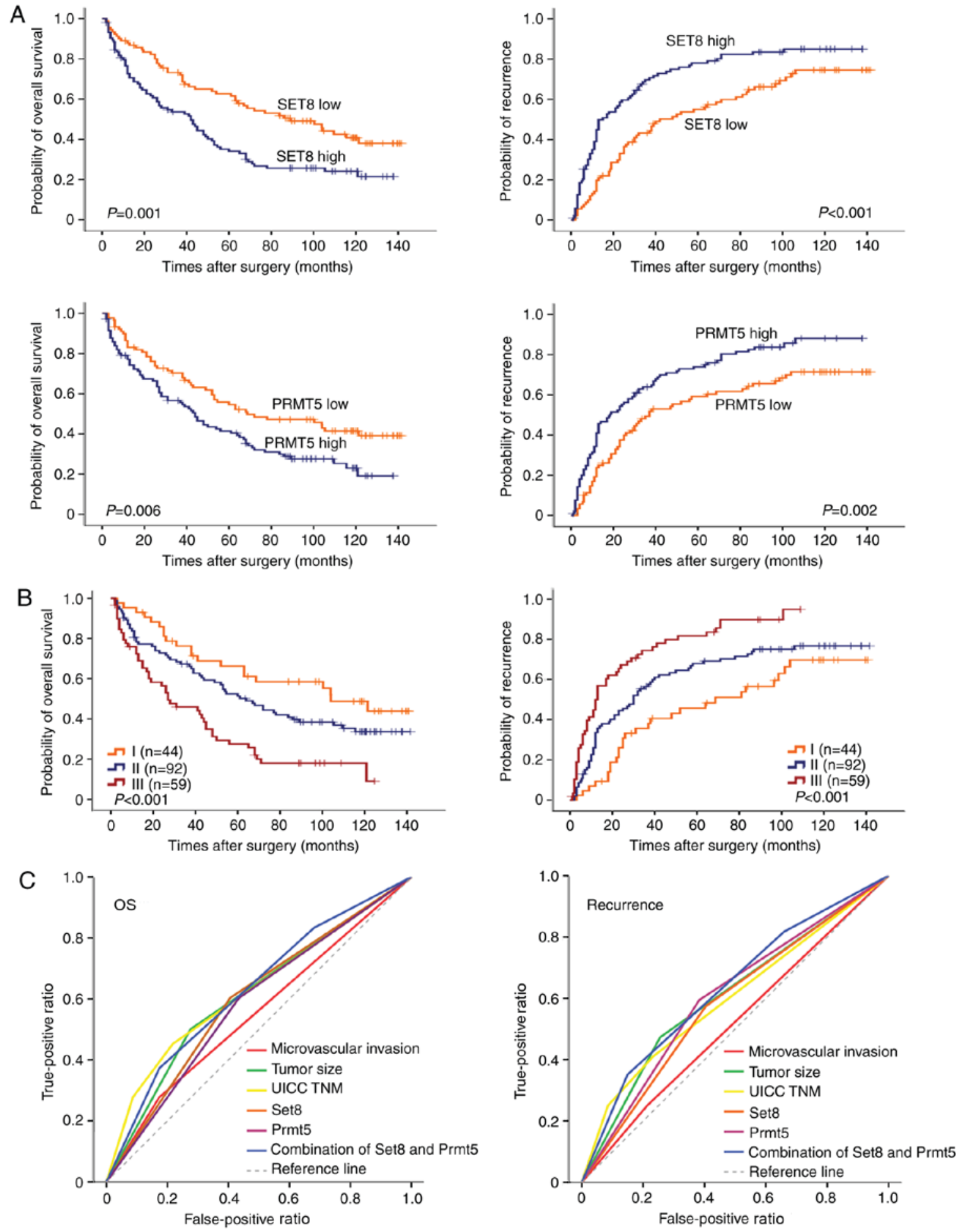

Figure 3. Prognostic and predictive value of SET8, PRMT5 and their combination in hepatocellular carcinoma. (A) Left panels; probability of overall survival over time following surgery for SET8 and PRMT5 low/high expression. Right panels; probability of recurrence over time following surgery for SET8 and PRMT5 low/high expression. (B) Cumulative overall survival (left panel) and time to recurrence (right panel) curves of the combination of SET8 and PRMT5 in patients with Tumor-Node-Metastasis I, II and III. Patients were classified into three groups according to their SET8 and PRMT5 expression: Group I, low expression of SET8 and PRMT5; group II, high expression of SET8 or PRMT5; and group III, high expression of SET8 and PRMT5. (C) Predictive value of clinicopathological variables adopted in receiver operating characteristic analysis for OS (left) and disease recurrence (right). SET8, SET domain-containing protein 8; PRMT5, protein arginine methyltransferase 5; UICC, International Union Against Cancer; TNM, tumor-node-metastasis.

distinct biological outcomes (23). Using high-throughput analyses, the statistical results of TMA staining in the results of the present study revealed that high expression of SET8 or PRMT5 was associated with OS and TTR following resection of a primary tumor, and the results of multivariate Cox proportional hazards analyses revealed that they were independent risk factors for OS and TTR. Furthermore, it was revealed that SET8 expression was positively associated with 
Table I. Association between clinicopathological features, SET8, PRMT5 expression and survival.

\begin{tabular}{|c|c|c|c|c|c|c|c|c|}
\hline \multirow[b]{3}{*}{ Factor } & \multicolumn{4}{|c|}{ OS } & \multicolumn{4}{|c|}{ TTR } \\
\hline & \multirow{2}{*}{$\begin{array}{c}\text { Univariate } \\
\mathrm{P} \text {-value }\end{array}$} & \multicolumn{3}{|c|}{ Multivariate } & \multirow{2}{*}{$\begin{array}{c}\text { Univariate } \\
\text { P-value }\end{array}$} & \multicolumn{3}{|c|}{ Multivariate } \\
\hline & & HR & $95 \% \mathrm{CI}$ & P-value & & HR & $95 \%$ CI & P-value \\
\hline Age ( $\leq 50$ vs. $>50$ years) & 0.126 & & & NA & 0.192 & & & NA \\
\hline Sex (female vs. male) & 0.105 & & & NA & 0.056 & & & NA \\
\hline ECOG (0 vs. 1$)$ & 0.39 & & & NA & 0.829 & & & NA \\
\hline Liver cirrhosis (yes vs. no) & 0.038 & 1.789 & $1.024-3.126$ & 0.041 & 0.05 & & & NS \\
\hline $\operatorname{AFP}(\leq 400$ vs. $>400 \mathrm{ng} / \mathrm{ml})$ & 0.029 & & & NS & 0.048 & & & NS \\
\hline Encapsulation (yes vs. no) & 0.284 & & & NA & 0.484 & & & NA \\
\hline Vascular invasion (yes vs. no) & $<0.001$ & 2.464 & $1.645-3.690$ & $<0.001$ & 0.006 & 1.783 & $1.216-2.614$ & 0.003 \\
\hline Tumor size $(\leq 5.8$ vs. $>5.8 \mathrm{~cm})$ & $<0.001$ & 1.956 & $1.358-2.817$ & $<0.001$ & $<0.001$ & 1.899 & $1.351-2.670$ & $<0.001$ \\
\hline Tumor number (single vs. multiple) & 0.002 & 1.613 & $1.053-2.471$ & 0.028 & 0.001 & 1.602 & $1.066-2.407$ & 0.023 \\
\hline UICC TNM stage (I vs. II vs. IIIA) & $<0.001$ & & & & $<0.001$ & & & \\
\hline Set8 (negative vs. positive) & 0.001 & 2.003 & $1.385-2.897$ & $<0.001$ & $<0.001$ & 1.894 & $1.356-2.646$ & $<0.001$ \\
\hline Prmt5 (negative vs. positive) & 0.006 & 1.712 & $1.176-2.492$ & 0.005 & 0.002 & 1.948 & $1.382-2.745$ & $<0.001$ \\
\hline Combine set 8 and Prmt5 & $<0.001$ & & & & $<0.001$ & 1.908 & $1.513-2.407$ & \\
\hline
\end{tabular}

OS, overall survival; TTR, time to recurrence; CI, confidence interval; HR, hazard ratio; NA, not adopted; NS, not significant; AFP, $\alpha$-fetoprotein; UICC, International Union Against Cancer; TNM, Tumor-Node-Metastasis; ECOG, Eastern Cooperative Oncology Group performance score; SET8, SET domain-containing protein 8; PRMT5, protein arginine methyltransferase 5.
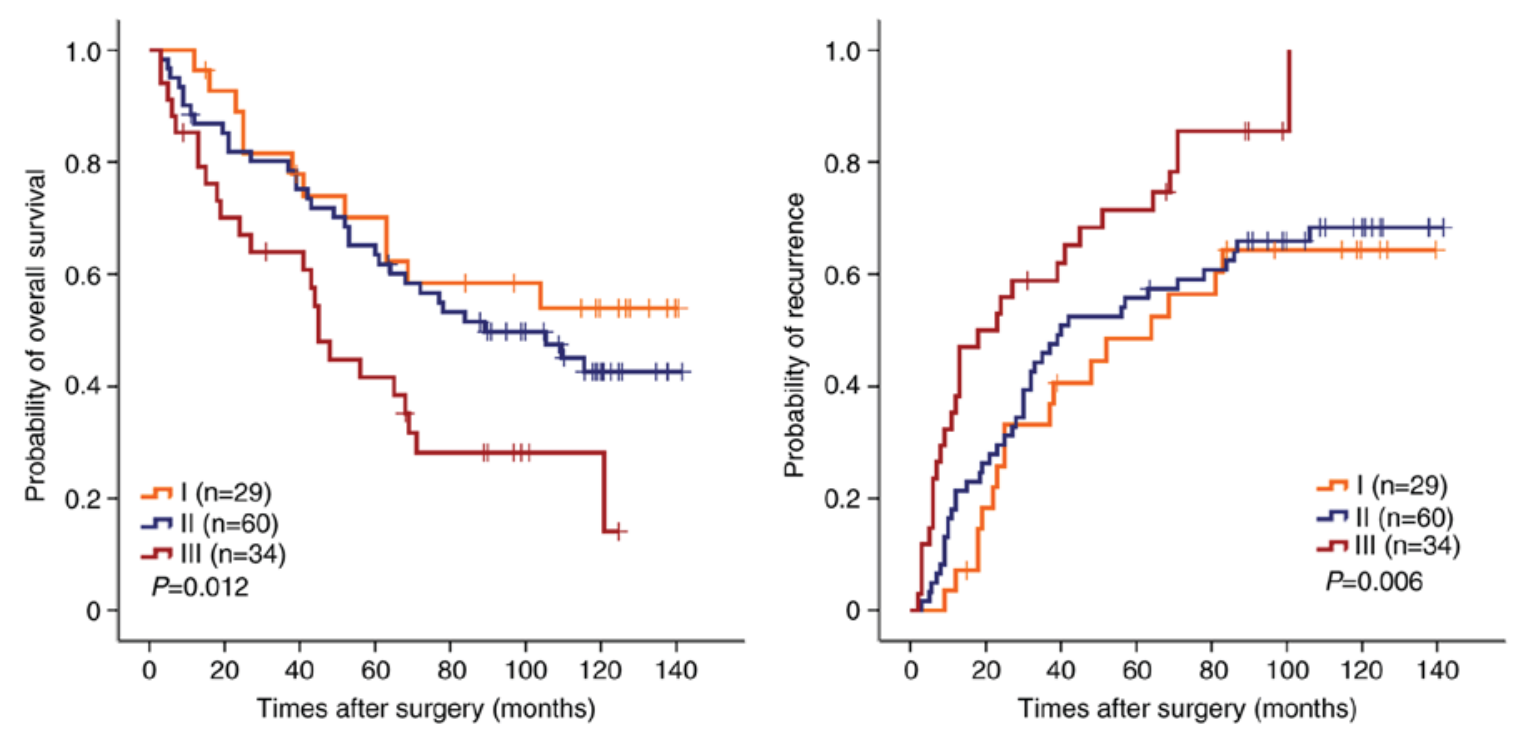

Figure 4. Kaplan-Meier curves of overall survival and disease recurrence (right panel) for expression of the combination of SET domain-containing protein 8 and protein arginine methyltransferase 5 in patients with Tumor-Node-Metastasis stage I.

PRMT5 expression, and the combination of SET8 and PRMT5 detection also improved the prognostic capacity. The results indicated that histone methylation in multiple sites within tumor tissue may have a synergistic effect on the progression of HCC.

Clinically, it remains difficult to predict the prognosis of patients with early stage HCC. In the present study, a significant trend of ER within 24 months after surgery was discovered in patients with high SET8 and PRMT5 expression. The combination of SET8 and PRMT5 expression could improve the predictive accuracy for OS and ER, which revealed the largest AUC for all clinicopathological variables investigated. Similarly, the prognostic value of SET8 and PRMT5 in those patients with early stage HCC (TNM stage I) and with a low AFP level ( $\leq 400 \mathrm{ng} / \mathrm{ml})$ was further validated. The OS and TTR rates of patients with early stage HCC or with low serum AFP and high SET8 and PRMT5 expression were significantly poorer than that of those with low expression 
A
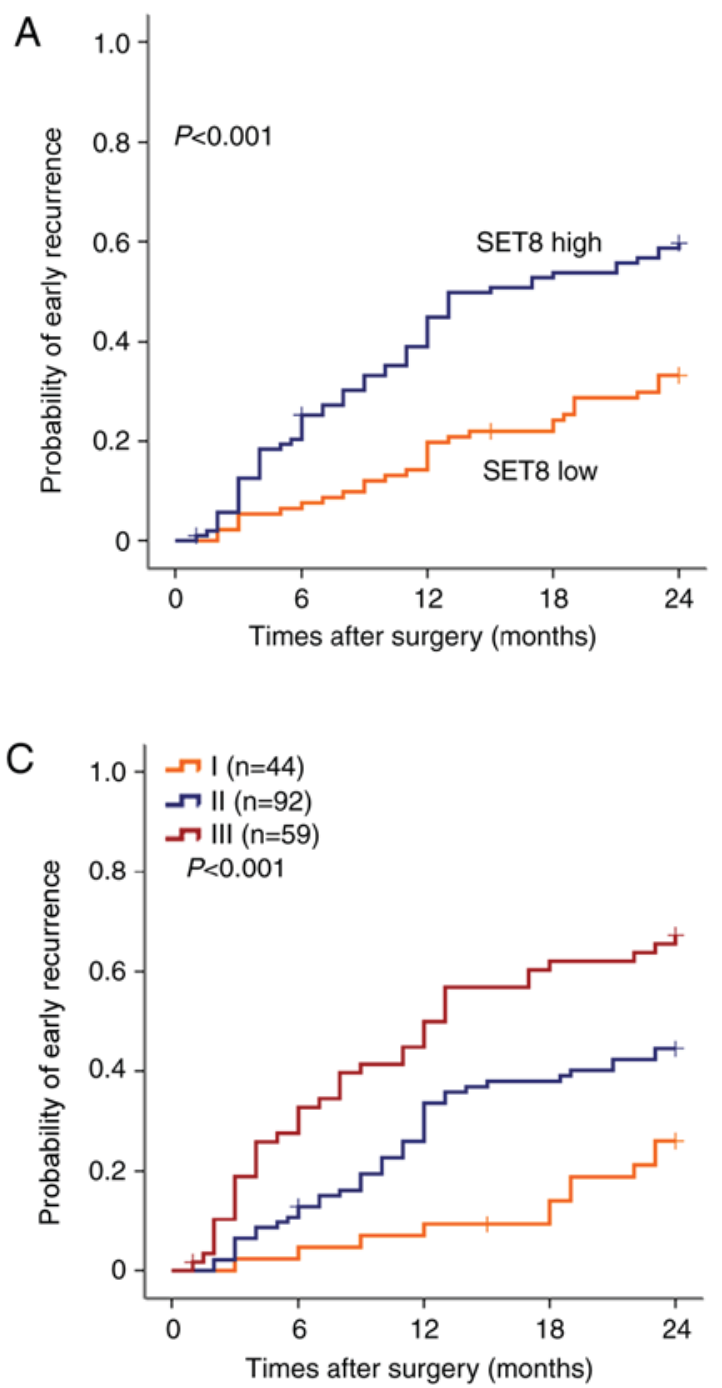

B
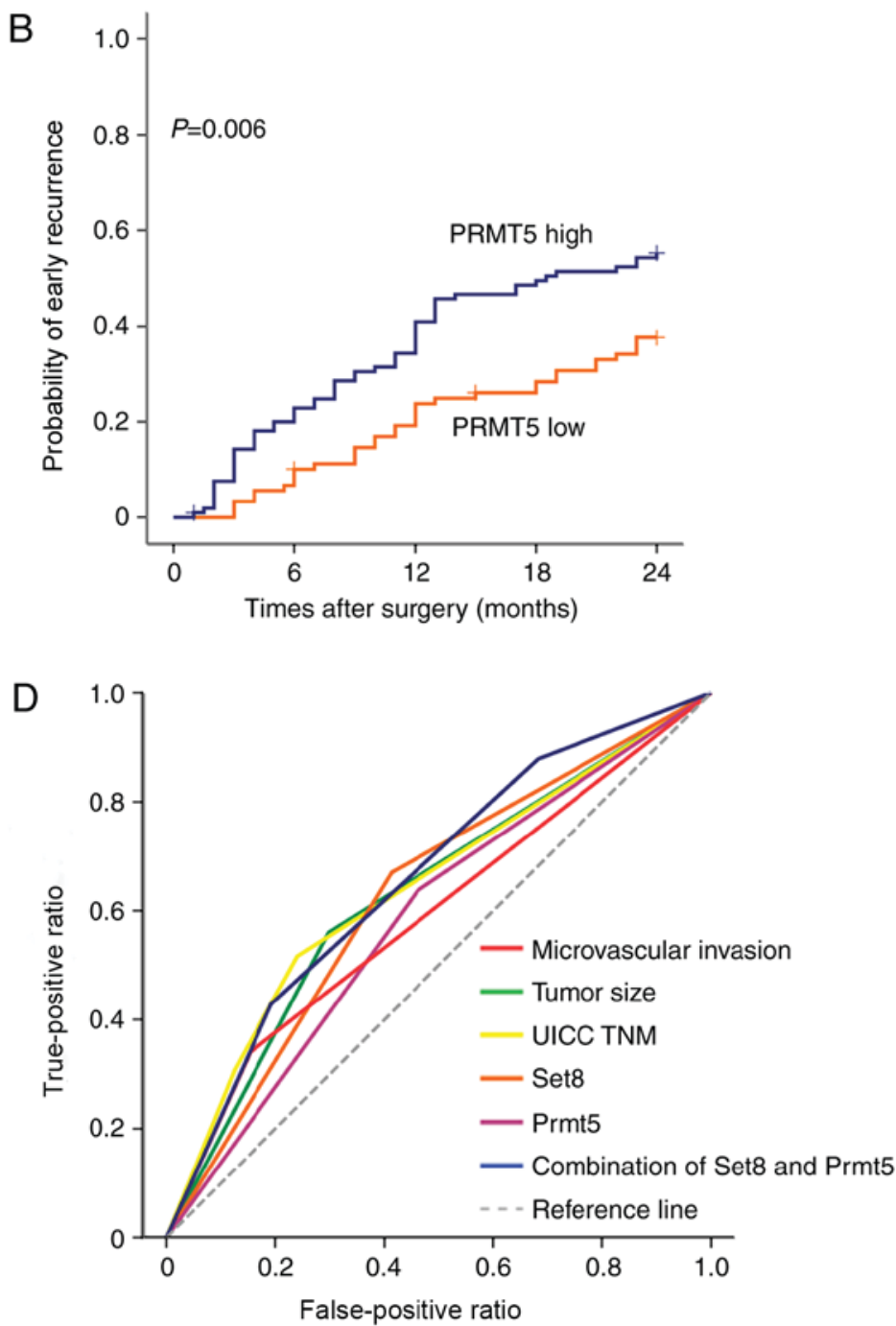

Figure 5. The predictive value of the expression of (A) SET domain-containing protein 8 (SET8) and (B) protein arginine methyltransferase 5 (PRMT5) for early recurrence ( $\leq 24$ months). (C) Cumulative time to early recurrence curves of the combination of SET8 and PRMT5 in patients with early recurrence. Patients were classified into three groups according to their SET8 and PRMT5 expression: Group I, low expression of SET8 and PRMT5; group II, high expression of SET8 or PRMT5; and group III, high expression of SET8 and PRMT5. (D) Predictive value of clinicopathological variables adopted in receiver operating characteristic analysis for early recurrence. SET8, SET domain-containing protein 8; PRMT5, protein arginine methyltransferase 5; UICC, International Union Against Cancer; TNM, Tumor-Node-Metastasis.

of the two. Therefore, more curative therapy, including liver transplantation, and a close follow-up should be considered in these high-risk patients.

The histone methyltransferases can not only serve as a prognostic tool but may also have a therapeutic role associated with inhibitors to enzymes that regulate histone methylation. A number of studies have focused on molecule targeting inhibitors of histone methyltransferase (31). For example, arginine methyltransferase inhibitor 1 (AMI-1) was generally considered to be a selective small-molecule inhibitor of type I PRMTs, while a previous study found it could inhibit HCC growth through inhibiting PRMT5. It was also indicated that PRMT5 is a potential pharmacologic therapeutic target for HCC (32). The present study demonstrated that the expression level of SET8 and PRMT5 was not correlated with hepatitis-related variables (serum ALT, presence of hepatitis B antigen or cirrhosis), or the tumor size or number. Therefore, it could be possible that the baseline expression level of histone methyltransferase is associated with specific heterogeneity among different individuals, including peritumoral environment. Due to the retrospective nature of the present study, the molecular mechanisms of the role of the peritumoral environment on HCC-associated histone methyltransferase was not addressed, and a selection bias may be present. However, postoperative adjuvant therapies targeting residual tumor cells and peritumoral environment may obtain improved therapeutic effects. Inhibiting multiple sites of histone methylation may be a novel therapeutic strategy for HCC.

In conclusion, high expression of SET8 combined with PRMT5 was correlated with high recurrence and poor OS rates in patients with HCC. The methylation of lysine and arginine in the tumor tissue represents a novel insight into tumor progression and recurrence, and therapeutic targets for HCC.

\section{Acknowledgements}

Not applicable. 


\section{Funding}

The present study was jointly supported by three grants (grant nos. 81672848 and 81372654) from the National Natural Science Foundation of China, and China National Science and Technology Major Project for Prevention and Treatment of Infectious Diseases (grant no. 2017ZX10203207).

\section{Availability of data and materials}

The datasets analyzed during the current study are available from the corresponding author on reasonable request.

\section{Authors' contributions}

$\mathrm{JZ}$ and JC designed and funded the project. ZL, HJ and $\mathrm{LH}$ conducted the experiments. ZL and JZ wrote the manuscript. YZ, WS, XR helped to collect the data from the patients. WZ, LL and ML analyzed the data. All authors read and approved the manuscript and agree to be accountable for all aspects of the research in ensuring that the accuracy or integrity of any part of the study are appropriately investigated and resolved.

\section{Ethics approval and consent to participate}

This retrospective study was carried out in accordance with the ethical standards of the institutional research committee and with the 1964 Helsinki declaration and its later amendments or ethical standards. The requirement to obtain informed consent was waived as this was a retrospective study. All patient data were treated in accordance with the local privacy regulations.

\section{Consent for publication}

Not applicable.

\section{Competing interests}

The authors declare that they have no competing interests.

\section{References}

1. Liu M, Jiang L and Guan XY: The genetic and epigenetic alterations in human hepatocellular carcinoma: A recent update. Protein Cell 5: 673-691, 2014.

2. Ma L, Chua MS, Andrisani O and So S: Epigenetics in hepatocellular carcinoma: An update and future therapy perspectives. World J Gastroenterol 20: 333-345, 2014.

3. Herath NI, Leggett BA and MacDonald GA: Review of genetic and epigenetic alterations in hepatocarcinogenesis. J Gastroenterol Hepatol 21: 15-21, 2006.

4. Wahid B, Ali A, Rafique S and Idrees M: New insights into the epigenetics of hepatocellular carcinoma. Biomed Res Int 2017: 1609575, 2017.

5. Pogribny IP and Rusyn I: Role of epigenetic aberrations in the development and progression of human hepatocellular carcinoma. Cancer Lett 342: 223-230, 2014.

6. Fang J, Feng Q, Ketel CS, Wang H, Cao R, Xia L, Erdjument-Bromage $\mathrm{H}$, Tempst P, Simon JA and Zhang Y: Purification and functional characterization of SET8, a nucleosomal histone $\mathrm{H} 4$-lysine 20-specific methyltransferase. Curr Biol 12: 1086-1099, 2002.

7. Xiao B, Jing C, Kelly G, Walker PA, Muskett FW, Frenkiel TA, Martin SR, Sarma K, Reinberg D, Gamblin SJ and Wilson JR: Specificity and mechanism of the histone methyltransferase Pr-Set7. Genes Dev 19: 1444-1454, 2005.
8. Nishioka K, Rice JC, Sarma K, Erdjument-Bromage H, Werner J, Wang Y, Chuikov S, Valenzuela P, Tempst P, Steward R, et al: PR-Set7 is a nucleosome-specific methyltransferase that modifies lysine 20 of histone $\mathrm{H} 4$ and is associated with silent chromatin. Mol Cell 9: 1201-1213, 2002.

9. Houston SI, McManus KJ, Adams MM, Sims JK, Carpenter PB, Hendzel MJ and Rice JC: Catalytic function of the PR-Set7 histone $\mathrm{H} 4$ lysine 20 monomethyltransferase is essential for mitotic entry and genomic stability. J Biol Chem 283: 19478-19488, 2008.

10. Oda H, Okamoto I, Murphy N, Chu J, Price SM, Shen MM, Torres-Padilla ME, Heard E and Reinberg D: Monomethylation of histone H4-lysine 20 is involved in chromosome structure and stability and is essential for mouse development. Mol Cell Biol 29: 2278-2295, 2009.

11. Li Z, Nie F, Wang S and Li L: Histone H4 Lys 20 monomethylation by histone methylase SET8 mediates Wnt target gene activation. Proc Natl Acad Sci USA 108: 3116-3123, 2011.

12. Wu S, Wang W, Kong X, Congdon LM, Yokomori K, Kirschner MW and Rice JC: Dynamic regulation of the PR-Set7 histone methyltransferase is required for normal cell cycle progression. Genes Dev 24: 2531-2542, 2010.

13. Jørgensen S, Elvers I, Trelle MB, Menzel T, Eskildsen M, Jensen ON, Helleday T, Helin K and Sørensen CS: The histone methyltransferase SET8 is required for S-phase progression. J Cell Biol 179: 1337-1345, 2007.

14. Congdon LM, Houston SI, Veerappan CS, Spektor TM and Rice JC: PR-Set7-mediated monomethylation of histone H4 lysine 20 at specific genomic regions induces transcriptional repression. J Cell Biochem 110: 609-619, 2010.

15. Abbas T, Shibata E, Park J, Jha S, Karnani N and Dutta A: CRL4(Cdt2) regulates cell proliferation and histone gene expression by targeting PR-Set7/Set 8 for degradation. Mol Cell 40: 9-21, 2010.

16. Stopa N, Krebs JE and Shechter D: The PRMT5 arginine methyltransferase: Many roles in development, cancer and beyond. Cell Mol Life Sci 72: 2041-2059, 2015.

17. Yang $\mathrm{Y}$ and Bedford MT: Protein arginine methyltransferases and cancer. Nat Rev Cancer 13: 37-50, 2013.

18. Pal S, Vishwanath SN, Erdjument-Bromage H, Tempst $\mathrm{P}$ and Sif S: Human SWI/SNF-associated PRMT5 methylates histone $\mathrm{H} 3$ arginine 8 and negatively regulates expression of ST7 and NM23 tumor suppressor genes. Mol Cell Biol 24: 9630-9645, 2004.

19. Pal S, Baiocchi RA, Byrd JC, Grever MR, Jacob ST and Sif S: Low levels of miR-92b/96 induce PRMT5 translation and H3R8/H4R3 methylation in mantle cell lymphoma. EMBO J 26: 3558-3569, 2007

20. Karkhanis V, Hu YJ, Baiocchi RA, Imbalzano AN and Sif S: Versatility of PRMT5-induced methylation in growth control and development. Trends Biochem Sci 36: 633-641, 2011.

21. Wei TY, Juan CC, Hisa JY, Su LJ, Lee YC, Chou HY, Chen JM, Wu YC, Chiu SC, Hsu CP, et al: Protein arginine methyltransferase 5 is a potential oncoprotein that upregulates G1 cyclins/cyclin-dependent kinases and the phosphoinositide 3-kinase/AKT signaling cascade. Cancer Sci 103: 1640-1650, 2012.

22. Bernstein BE, Meissner A and Lander ES: The mammalian epigenome. Cell 128: 669-681, 2007.

23. Strahl BD and Allis CD: The language of covalent histone modifications. Nature 403: 41-45, 2000.

24. Zhang Y and Reinberg D: Transcription regulation by histone methylation: Interplay between different covalent modifications of the core histone tails. Genes Dev 15: 2343-2360, 2001.

25. Qian YB, Zhang JB, Wu WZ, Fang HB, Jia WD, Zhuang PY, Zhang $\mathrm{BH}$, Pan Q, Xu Y, Wang L, et al: $\mathrm{P} 48$ is a predictive marker for outcome of postoperative interferon-alpha treatment in patients with hepatitis B virus infection-related hepatocellular carcinoma. Cancer 107: 1562-1569, 2006.

26. Bruix J and Sherman M; Practice Guidelines Committee, American Association for the Study of Liver Diseases: Management of hepatocellular carcinoma. Hepatology 42: 1208-1236, 2005.

27. Imamura H, Matsuyama Y, Tanaka E, Ohkubo T, Hasegawa K, Miyagawa S, Sugawara Y, Minagawa M, Takayama T, Kawasaki S and Makuuchi M: Risk factors contributing to early and late phase intrahepatic recurrence of hepatocellular carcinoma after hepatectomy. J Hepatol 38: 200-207, 2003. 
28. Budhu A, Forgues M, Ye QH, Jia HL, He P, Zanetti KA, Kammula US, Chen Y, Qin LX, Tang ZY and Wang XW: Prediction of venous metastases, recurrence, and prognosis in hepatocellular carcinoma based on a unique immune response signature of the liver microenvironment. Cancer Cell 10: 99-111, 2006.

29. Seligson DB, Horvath S, Shi T, Yu H, Tze S, Grunstein M and Kurdistani SK: Global histone modification patterns predict risk of prostate cancer recurrence. Nature 435: 1262-1266, 2005.

30. Jenuwein $\mathrm{T}$ and Allis CD: Translating the histone code. Science 293: 1074-1080, 2001.
31. Cheng D, Yadav N, King RW, Swanson MS, Weinstein EJ and Bedford MT: Small molecule regulators of protein arginine methyltransferases. J Biol Chem 279: 23892-23899, 2004.

32. Zhang B, Dong S, Li Z, Lu L, Zhang S, Chen X, Cen X and Wu Y: Targeting protein arginine methyltransferase 5 inhibits human hepatocellular carcinoma growth via the downregulation of beta-catenin. J Transl Med 13: 349, 2015.

cc) (1) (9) This work is licensed under a Creative Commons cc) Attribution-NonCommercial-NoDerivatives 4.0 International (CC BY-NC-ND 4.0) License. 